\title{
DOA Estimation of a Novel Generalized Nested MIMO Radar with High Degrees of Freedom and Hole-Free Difference Coarray
}

\author{
Yule Zhang $\mathbb{D}^{1,2}$ Guoping $\mathrm{Hu} \mathbb{D}^{1,2}{ }^{2}$ Hao Zhou, ${ }^{2}$ Mingming Zhu, ${ }^{1,2}$ and Fei Zhang ${ }^{1,3}$ \\ ${ }^{1}$ Graduate College, Air Force Engineering University, Xi'an 710051, China \\ ${ }^{2}$ Air and Missile Defense College, Air Force Engineering University, Xi'an 710051, China \\ ${ }^{3}$ Aeronautics Engineering College, Air Force Engineering University, Xi'an 710051, China \\ Correspondence should be addressed to Yule Zhang; yule_zhang0921@163.com
}

Received 26 December 2020; Revised 8 January 2021; Accepted 12 January 2021; Published 27 January 2021

Academic Editor: Wang Zheng

Copyright ( 92021 Yule Zhang et al. This is an open access article distributed under the Creative Commons Attribution License, which permits unrestricted use, distribution, and reproduction in any medium, provided the original work is properly cited.

\begin{abstract}
A novel generalized nested multiple-input multiple-output (MIMO) radar for direction of arrival (DOA) estimation is proposed in this paper. The proposed structure utilizes the extended two-level nested array (ENA) as transmitter and receiver and adjusts the interelement spacing of the receiver with an expanding factor. By optimizing the array element configuration, we can obtain the best number of elements of the transmitter and receiver and the attainable degrees of freedom (DOF). Compared with the existing nested MIMO radar, the proposed MIMO array configuration not only has closed-form expressions for sensors' positions and the number of maximum DOF, but also significantly improves the array aperture. It is verified that the sum-difference coarray (SDCA) of the proposed nested MIMO radar can get higher DOF without holes. MUSIC algorithm based on Toeplitz matrix reconstruction is employed to prove the rationality and superiority of the proposed MIMO structure.
\end{abstract}

\section{Introduction}

Multiple-input multiple-output (MIMO) radar [1-3], with good space, frequency, and waveform diversity characteristics, is widely used in array signal processing [4-6] in the last few years. Compared with phased array radar, it has significant advantages in signal detection, parameter estimation [7], direction finding accuracy, spatial resolution [6], and antijamming capabilities, etc. However, the traditional MIMO radar usually adopts a uniform linear array (ULA) as transmitter and receiver, whose interelement spacing is equal to and no more than half wavelength. Hence, there are some problems in the direction of arrival (DOA) estimation for MIMO radar, such as mutual coupling of array elements [8] and limited aperture of virtual array elements [9].

In order to enhance the upper limit of degrees of freedom (DOF) and the flexibility of layout as well as reducing mutual coupling of physical sensors, sparse arrays such as the minimum redundancy array (MRA) $[10,11]$, coprime array (CPA) [12], and nested array (NA) [13-17] have been explored for DOA estimation and joint multiparameter estimation. In addition, sparse arrays combined with MIMO radar can further increase DOF through the sum-difference coarray (SDCA) [18], so as to improve the accuracy of direction finding and angle resolution ability.

For the purpose of improving sensor utilization, the minimum redundancy MIMO radar [19] designs the optimal array spacing by optimizing the number of virtual array elements, whereas it requires complex computational search and lacks closed-form expressions of DOF. The coprime MIMO radar generally uses part [20] or whole CPA [21] as the transmitting array and the receiving array. Li et al. [20] combined it with the real-value ESPRIT algorithm to DOA estimation, but did not consider the virtual array expansion of the echo signal model. Therefore, its DOF is limited by the number of physical sensors. Shi et al. [22] defined the generalized sum-difference coarray (GSDC) and simultaneously derived the closed-form expressions of the total number of virtual array elements of the generalized two-level coprime MIMO radar, which can obtain $O\left(M^{2} N^{2}\right)$ DOF by $O(M+N)$ physical sensors. The obtained DOF are much 
higher than that in $[20,21]$ with $O(M N)$ DOF. Unfortunately, there are holes in the SDCA of the abovementioned coprime MIMO radars, so multiple signal classification (MUSIC) [23] and estimation of signal parameters via rotational invariance technique (ESPRIT) algorithms [24] cannot be firsthand applied to these array structures.

Nested MIMO radar $[25,26]$ has closed-form expressions of positions and the number of virtual array elements and overcomes the defects of the minimum redundancy MIMO radar and coprime MIMO radar. Qin et al. [25] exploited nested subarrays as transmitting and receiving arrays to DOA estimation of mixed coherent and uncorrelated targets. Zheng et al. [26] adopted traditional twolevel nested MIMO array to joint direction of departure (DOD) and direction of arrival (DOA) estimation with closed-form DOF. They can provide $O\left(M^{2}\right)$ DOF with $O(M)$ sensors. Yang et al. $[27,28]$ designed a hole-free generalized nested MIMO configuration on the concept of the conventional two-level nested array, which improves DOF and angle estimation performance while effectively reducing the mutual coupling between the transmitting sensors. Specifically, it can provide $O\left(M^{4}\right)$ DOF with $O(M)$ elements.

To further enhance DOF, this paper adopts the extended two-level nested array (ENA) [15] to construct a new generalized nested MIMO radar. Firstly, the whole ENA is used as the transmitting array and receiving array of MIMO radar. Next, an expanding factor is employed to increase the receiving array spacing, and closed-form expressions of DOF and the best physical array element configuration are derived. Afterwards, Toeplitz matrix reconstruction [29] based on the MUSIC algorithm is employed to exploit the superiority of the proposed array configuration.

To be more specific, the main contributions of this paper are as follows:

(a) The optimal array element configuration structure of ENA is deduced and higher degrees of freedom are obtained. Besides, the difference coarray (DCA) is a ULA without holes.

(b) A new generalized nested MIMO radar based on ENA is constructed, and the optimal sensors' positions and the maximum DOF are derived, which can obtain $O\left(G^{4}\right)$ DOF with $O(G)$ sensors. Meanwhile, the SDCA is a ULA without holes. Its DOF is much higher than the existing nested MIMO radars in [25-28].

\section{Echo Signal Model}

A monostatic sparse array MIMO radar consists of a transmitter with $M=M_{1}+M_{2}$ arrays and a receiver with $N=N_{1}+N_{2}$ arrays. The positions of the transmitting array are located at $\mathbf{P}_{t}=\left\{p_{t m} \mid m=1,2, \ldots, M\right\}$ and the positions of the receiving array are located at $\mathbf{P}_{r}=\left\{p_{r n} \mid n=1,2, \ldots, N\right\}$, respectively. The unit array element spacing $d$ of the sensor is equal to $\lambda / 2$, where $\lambda$ stands for the signal wavelength. Suppose that there are $K$ far-field uncorrelated narrowband sources from angles $\theta=\left\{\theta_{k} \mid k=1,2, \ldots, K\right\}$, and the reflection coefficient of the $k$-th source is $\beta_{k}$. Then, the echo signal model can be expressed as follows:

$$
\mathbf{x}(t)=\sum_{k=1}^{K} \boldsymbol{\alpha}_{r}\left(\theta_{k}\right) \beta_{k} \boldsymbol{\alpha}_{t}^{T}\left(\theta_{k}\right) \mathbf{b}(t)+\mathbf{w}(t),
$$

where $\mathbf{b}(t)=\left[b_{0}(t), b_{1}(t), \ldots, b_{M-1}(t)\right]^{T}$ denotes the transmit signal; $\mathbf{w}(t)$ is an additive white Gaussian noise; and $\alpha_{t}\left(\theta_{k}\right)$ and $\alpha_{r}\left(\theta_{k}\right)$ are the transmit steering vectors and receive steering vectors of the $k$-th source, respectively, which can be expressed as

$$
\begin{aligned}
& \boldsymbol{\alpha}_{t}\left(\theta_{k}\right)=\left[1, e^{\left(-j 2 \pi p_{t 2} \sin \theta_{k} / \lambda\right)}, \ldots, e^{\left(-j 2 \pi p_{t M} \sin \theta_{k} / \lambda\right)}\right]^{T}, \\
& \boldsymbol{\alpha}_{r}\left(\theta_{k}\right)=\left[1, e^{\left(-j 2 \pi p_{r 2} \sin \theta_{k} / \lambda\right)}, \ldots, e^{\left(-j 2 \pi p_{r N} \sin \theta_{k} / \lambda\right)}\right]^{T},
\end{aligned}
$$

where $p_{t m} \in \mathbf{P}_{t}$ and $p_{r n} \in \mathbf{P}_{r}$ represent the sensor positions in the transmitter and receiver, respectively, and $p_{t 1}=p_{r 1}=0$.

Since the transmitting waveforms of MIMO radar are orthogonal to each other, i.e., $\mathbf{R}_{b}=E\left[\mathbf{b}(t) \mathbf{b}(t)^{H}\right]=\mathbf{I}_{M \times N}$, the output of the generalized matched filters for the echo signal can be expressed as follows:

$$
\begin{aligned}
x(t) & =\sum_{k=1}^{K} \beta_{k}\left(\boldsymbol{a}_{t}\left(\theta_{k}\right) \otimes \boldsymbol{a}_{r}\left(\theta_{k}\right)\right)+\mathbf{n}(t) \\
& =\left[\boldsymbol{a}_{t}\left(\theta_{1}\right) \otimes \boldsymbol{a}_{r}\left(\theta_{1}\right), \ldots, \boldsymbol{a}_{t}\left(\theta_{K}\right) \otimes \boldsymbol{\alpha}_{r}\left(\theta_{K}\right)\right] \mathbf{s}(t)+\mathbf{n}(t) \\
& =\left(\mathbf{A}_{t} \odot \mathbf{A}_{r}\right) \mathbf{s}(t)+\mathbf{n}(t) .
\end{aligned}
$$

where $\mathbf{A}_{t}=\left[\mathbf{a}_{t}\left(\theta_{1}\right), \mathbf{a}_{t}\left(\theta_{2}\right), \ldots, \mathbf{a}_{t}\left(\theta_{K}\right)\right] ; \quad \mathbf{A}_{r}=\left[\mathbf{a}_{r}\left(\theta_{1}\right), \mathbf{a}_{r}\right.$ $\left.\left(\theta_{2}\right), \ldots, \mathbf{a}_{r}\left(\theta_{K}\right)\right] ; \mathbf{n}(t)$ is an additive white Gaussian noise vector; $\mathbf{s}(t)=\left[\beta_{1}, \beta_{2}, \ldots, \beta_{K}\right]^{T} ; \otimes$ and $\odot$ denote Kronecker product and Khatri-Rao product, respectively.

The covariance matrix of the echo signal can be obtained by

$$
\begin{aligned}
\mathbf{R} & =E\left[\mathbf{x}(t) \mathbf{x}(t)^{H}\right]=\left(\mathbf{A}_{t} \odot \mathbf{A}_{r}\right) \mathbf{R}_{s}\left(\mathbf{A}_{t} \odot \mathbf{A}_{r}\right)^{H}+\sigma_{n}^{2} \mathbf{I}_{M_{t} N_{r}} \\
& =\mathbf{A R}_{s} \mathbf{A}^{H}+\sigma_{n}^{2} \mathbf{I}_{M_{t} N_{r}},
\end{aligned}
$$

where $\mathbf{R}_{s}=E\left[s(t) s(t)^{H}\right]=\operatorname{diag}\left[\sigma_{1}^{2}, \sigma_{2}^{2}, \ldots, \sigma_{k}^{2}\right]$ is the target covariance matrix, $\sigma_{k}^{2}$ denotes the signal energy of the $k$-th target, $\mathbf{A}=\mathbf{A}_{t} \odot \mathbf{A}_{r}$, and $\sigma_{n}^{2}$ is the noise variance.

The observing vector can be obtained by vectorizing $\mathbf{R}$ :

$$
\begin{aligned}
r & =\operatorname{vec}(\mathbf{R})=\left(\mathbf{A}^{*} \odot \mathbf{A}\right) p+\sigma_{n}^{2} \operatorname{vec}\left(\mathbf{I}_{M_{t} N_{r}}\right) \\
& =\left(\mathbf{A}^{*} \odot \mathbf{A}\right) p+\sigma_{n}^{2} \operatorname{vec}\left(\mathbf{I}_{M_{t} N_{r}}\right)=\mathbf{B} p+\sigma_{n}^{2} \operatorname{vec}\left(\mathbf{I}_{M_{t} N_{r}}\right),
\end{aligned}
$$

where $\operatorname{vec}(\cdot)$ represents vectorized operation; $\mathbf{p}=\left[\sigma_{1}^{2}, \sigma_{2}^{2}, \ldots, \sigma_{k}^{2}\right]^{T} ;(\cdot)$ implies the complex conjugation of the matrix. 


$$
\begin{aligned}
\mathbf{B}= & \mathbf{A}^{*} \odot \mathbf{A}=\left[\mathbf{a}_{t}^{*}\left(\theta_{1}\right) \otimes \mathbf{a}_{r}^{*}\left(\theta_{1}\right) \otimes \mathbf{a}_{t}\left(\theta_{1}\right) \otimes \mathbf{a}_{r}\left(\theta_{1}\right), \ldots, \mathbf{a}_{t}^{*}\left(\theta_{k}\right)\right. \\
& \left.\otimes \mathbf{a}_{r}^{*}\left(\theta_{k}\right) \otimes \mathbf{a}_{t}\left(\theta_{k}\right) \otimes \mathbf{a}_{r}\left(\theta_{k}\right)\right] .
\end{aligned}
$$

\section{Extended Two-level Nested Array}

In this section, the configuration of the extended two-level nested array (ENA) is formulated first. Then the optimal configuration structure of the physical array elements and the closed-form expressions of its DOF are derived.

3.1. ENA Configuration. As shown in Figure 1, the ENA configuration maintains the basic structure of the nested array, except for increasing the first two sensors of the sparse ULA by $d$ intervals. And the total number of sensors is equal to $M=M_{1}+M_{2}$.

Therefore, the extended two-level nested array sensor location can be expressed as

$$
\begin{aligned}
\mathbf{P}_{\mathrm{ENA}}= & \left\{0,1, \ldots, M_{1}-1, M, 2\left(M_{1}+1\right), 3\left(M_{1}+1\right),\right. \\
& \left.\ldots, M_{2}\left(M_{1}+1\right)\right\} .
\end{aligned}
$$

Then, the difference coarray of ENA can be defined as

$$
\begin{aligned}
\mathbf{S}_{\mathrm{ENA}}=\left\{s-\widetilde{s}, s, \widetilde{s} \in \mathbf{P}_{\mathrm{ENA}}\right\}=\left\{-M_{2}\left(M_{1}+1\right),\right. \\
\\
\left.\ldots, 0, \ldots, M_{2}\left(M_{1}+1\right)\right\} .
\end{aligned}
$$

3.2. Attainable DOF of ENA. According to equation (8), the DOF of ENA is

$$
\mathrm{DOF}=2 M_{2}\left(M_{1}+1\right)+1 .
$$

When the total number of sensors is fixed to $M=M_{1}+M_{2}$, the array element optimal configuration structure can be optimized to have attainable DOF, as shown in Table 1.

\section{Proposed Generalized Nested MIMO Radar}

In this section, the entire ENA is adopted as the transmitter and receiver to construct an extended nested MIMO radar (ENA-TR). Furthermore, a generalized extended two-level nested MIMO radar (GENA-TR) based on ENA-TR is proposed and the closed-form expressions for attainable DOF are deduced.

4.1. ENA-TR. From equation (7), the position of the transmitter with $M$ sensors and receiver with $N$ sensors is given by

$$
\begin{aligned}
& \mathbf{P}_{T}=\left\{0,1, \ldots, M_{1}-1, M_{1}, 2\left(M_{1}+1\right), 3\left(M_{1}+1\right),\right. \\
&\left.\ldots, M_{2}\left(M_{1}+1\right)\right\}, \\
& \mathbf{P}_{R}=\left\{0,1, \ldots, N_{1}-1, N_{1}, 2\left(N_{1}+1\right), 3\left(N_{1}+1\right),\right. \\
&\left.\ldots, N_{2}\left(N_{1}+1\right)\right\} .
\end{aligned}
$$

According to equation (8), the difference coarray of the transmitter and receiver are both consecutive ULAs with $2 M_{2}\left(M_{1}+1\right)+1$ and $2 N_{2}\left(N_{1}+1\right)+1$ virtual array elements, which are located at

$$
\begin{aligned}
\mathbf{S}_{T}= & \left\{s_{t}-s_{\tilde{t}}, s_{t}, s_{t} \in \mathbf{P}_{T}\right\}=\left\{-M_{2}\left(M_{1}+1\right),\right. \\
& \left.\ldots, 0, \ldots M_{2}\left(M_{1}+1\right)\right\}, \\
\mathbf{S}_{R}= & \left\{s_{r}-s_{\widetilde{r}}, s_{r}, s_{r} \in \mathbf{P}_{R}\right\}=\left\{-N_{2}\left(N_{1}+1\right),\right. \\
& \left.\ldots, 0, \ldots N_{2}\left(N_{1}+1\right)\right\} .
\end{aligned}
$$

It can be known from equation (6) that the virtual element positions of $\mathbf{B}=\mathbf{A}^{*} \odot \mathbf{A}$ are composed of sum-difference coarray of physical sensor positions.

$$
\begin{aligned}
\mathbf{S}_{\mathrm{SDCA}}^{\mathrm{ENA}} & =\left\{\left(s_{t}+s_{r}\right)-\left(s_{\widetilde{t}}+s_{\widetilde{r}}\right) \mid s_{t}, s_{\tilde{t}} \in \mathbf{P}_{T}, s_{r}, s_{\widetilde{r}} \in \mathbf{P}_{R}\right\} \\
& =\left\{\left(s_{t}+s_{\tilde{t}}\right)-\left(s_{r}+s_{\widetilde{r}}\right) \mid s_{t}, s_{\widetilde{t}} \in \mathbf{P}_{T}, s_{r}, s_{\widetilde{r}} \in \mathbf{P}_{R}\right\} \\
& =\left\{l_{t}+l_{r} \mid l_{t} \in \mathbf{S}_{T}, l_{r} \in \mathbf{S}_{R}\right\} .
\end{aligned}
$$

Therefore, the sum-difference coarray of ENA-TR is essentially the sum coarray of two difference coarrays.

4.2. GENA-TR. The ENA-TR configuration fails to make full use of the virtual aperture expansion effect of the sumdifference coarray. By introducing the interelement spacing expansion factor, a generalized ENA-TR (GENA-TR) is established to increase DOF, as shown in Figure 2.

The interelement spacing of the receiver is enlarged with an expansion factor $\alpha$, so the receiver sensor positions are located at

$$
\begin{aligned}
\mathbf{P}_{R}^{\alpha}= & \alpha \mathbf{P}_{R}=\alpha\left\{0,1, \ldots, N_{1}-1, N_{1}, 2\left(N_{1}+1\right), 3\left(N_{1}+1\right),\right. \\
& \left.\ldots, N_{2}\left(N_{1}+1\right)\right\} .
\end{aligned}
$$

It can be seen from set (15) that the difference coarray of the receiver is a filled ULA with interelement spacing enlarged by factor $\alpha$. And the difference coarray set is given by

$$
\begin{aligned}
\mathbf{S}_{R}^{\alpha}= & \left\{s_{r}^{\alpha}-s_{r}^{\alpha}, s_{r}^{\alpha}, s_{r}^{\alpha} \in \mathbf{P}_{r}^{\alpha}\right\}=\alpha\left\{-N_{2}\left(N_{1}+1\right), \ldots, 0,\right. \\
& \left.\ldots, N_{2}\left(N_{1}+1\right)\right\} .
\end{aligned}
$$

According to equations (10) and (15), we can get the sum-difference coarray set of GENA-TR:

$$
\begin{aligned}
\mathbf{S}_{\mathrm{SDCA}}^{\mathrm{GENA}-\mathrm{TR}} & =\left\{\left(s_{t}+s_{r}^{\alpha}\right)-\left(s_{t}+s_{r}^{\alpha}\right), s_{t}, s_{\bar{t}} \in \mathbf{P}_{T}, s_{r}^{\alpha}, s_{r}^{\alpha} \in \mathbf{P}_{R}^{\alpha}\right\} \\
& =\left\{\left(s_{t}+s_{t}\right)-\left(s_{r}^{\alpha}+s_{r}^{\alpha}\right), s_{t}, s_{t} \in \mathbf{P}_{T}, s_{r}^{\alpha}, s_{r}^{\alpha} \in \mathbf{P}_{R}^{\alpha}\right\} \\
& =\left\{l_{t}+l_{r}^{\alpha}, l_{t} \in \mathbf{S}_{T}, l_{r}^{\alpha}, \in \mathbf{S}_{R}^{\alpha}\right\} .
\end{aligned}
$$

Proposition 1. The sum-difference coarray of GENA-TR has the following properties:

(a) The range of the expansion factor $\alpha$ is $1 \leq \alpha \leq 2 M_{2}\left(M_{1}+1\right)+1$ 


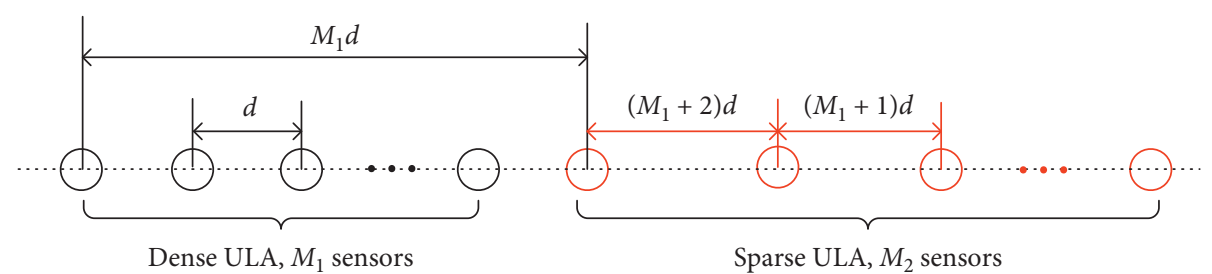

FIgURE 1: ENA configuration.

TABle 1: Optimal configuration structure for ENA.

\begin{tabular}{lcr}
\hline$M$ & Optimal $M_{1}, M_{2}$ & DOF \\
\hline Odd & $M_{1}=(M-1) / 2, M_{2}=(M+1) / 2$ & $\left(M^{2}+3\right) / 2+M$ \\
Even & $M_{1}=M_{2}=M / 2$ & $\left(M^{2}+2\right) / 2+M$ \\
\hline
\end{tabular}
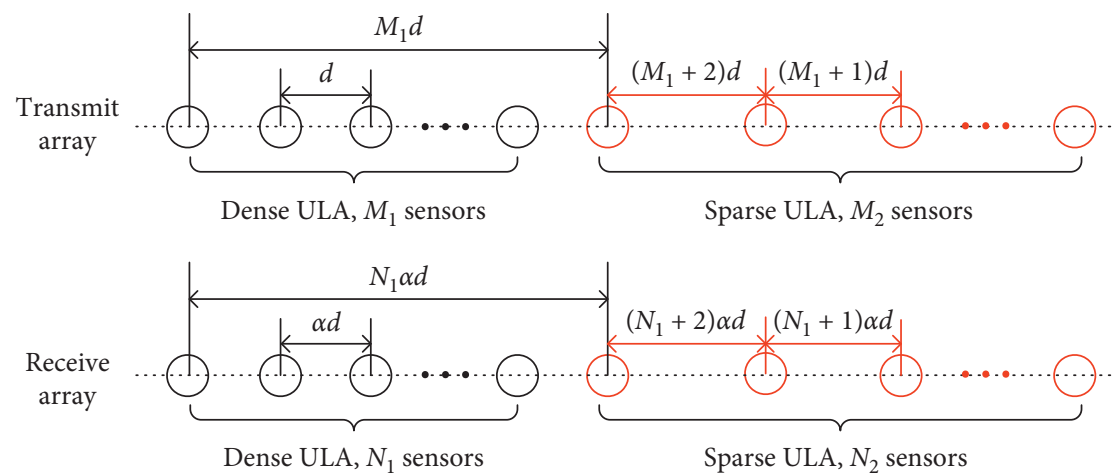

FIGURE 2: GENA-TR structure.

(b) $\mathbf{S}_{S D C A}^{G E N A-T R}$ contains all the consecutive integers in the range $[-V, V]$, where $V=M_{2}\left(M_{1}+1\right)+$ $\alpha N_{2}\left(N_{1}+1\right)$

(c) The sum-difference coarray of GENA-TR contains $2\left[M_{2}\left(M_{1}+1\right)+2 \alpha N_{2}\left(N_{1}+1\right)\right]+1$ unique lags of virtual array elements without holes

Proof.

(a) It can be known from equations (12) and (16) that $\mathbf{S}_{T}$ and $\mathbf{S}_{R}^{\alpha}$ are symmetrical sets, so $l_{t}+l_{r}^{\alpha}=l_{t}-l_{r}^{\alpha}$. Therefore, the sum-difference coarray of GENA-TR is essentially a difference coarray of two subarrays. When $1 \leq \alpha \leq 2 M_{2}\left(M_{1}+1\right)+1, \mathbf{S}_{\mathrm{SDCA}}^{\mathrm{GENA}-\mathrm{TR}}$ can get consecutive virtual array elements with certain positions [13].

(b) According to equations (12) and (16), the margins of $\mathbf{S}_{\mathrm{SDCA}}^{\mathrm{GENA}-\mathrm{TR}}$ can be obtained.

For the left margin,

$$
-V=-M_{2}\left(M_{1}+1\right)+\left[-\alpha N_{2}\left(N_{1}+1\right)\right] .
$$

For the right margin,

$$
V=M_{2}\left(M_{1}+1\right)+\left[\alpha N_{2}\left(N_{1}+1\right)\right] \text {. }
$$

In addition, $\mathbf{S}_{T}$ and $\mathbf{S}_{R}^{\alpha}$ are both consecutive integers, so $S_{\text {SDCA }}^{\mathrm{GENA}}$ is a hole-free ULA.

(c) According to proposition (b), the maximum number of $\mathbf{S}_{\mathrm{SDCA}}^{\mathrm{GENA}-\mathrm{TR}}$ is $2 \mathrm{~V}+1=2\left[M_{2}\left(M_{1}+1\right)+\right.$ $\left.2 \alpha N_{2}\left(N_{1}+1\right)+1\right]$.

The sum-difference coarray of GENA-TR can attain the maximum number of DOF, where $\alpha=2 M_{2}\left(M_{1}+1\right)+1$. When the total number of physical array elements is determined to be $G$, the array construction problem turns to an optimization problem about the maximum number of DOF

$$
\begin{aligned}
& \max \left[2 M_{2}\left(M_{1}+1\right)+1\right]\left[2 N_{2}\left(N_{1}+1\right)+1\right] \\
& \text { s.t. } G=M_{1}+M_{2}+N_{1}+N_{2} .
\end{aligned}
$$

The Lagrange function of equation (20) can be expressed as

$$
\begin{aligned}
f= & {\left[2 M_{2}\left(M_{1}+1\right)+1\right]\left[2 N_{2}\left(N_{1}+1\right)\right] } \\
& +\eta\left(M_{1}+M_{2}+N_{1}+N_{2}-G\right) .
\end{aligned}
$$

where $\eta$ represents the Lagrange multiplier.

Taking the partial of $f$ with respect to $M_{1}, M_{2}, N_{1}, N_{2}, \eta$, the following system of equations can be expressed: 
TABLE 2: Optimal configuration structure for GENA-TR.

\begin{tabular}{|c|c|c|}
\hline$G$ & Optimal $M_{1}, M_{2}, N_{1}, N_{2}$ & DOF \\
\hline$G=4 k$ & $M_{1}=M_{2}=N_{1}=N_{2}=G / 4$ & $\left(G^{4}+8 G^{3}+32 G^{2}+64 G+64\right) / 64$ \\
\hline$G=4 k+1$ & $\begin{array}{l}M_{1}=N_{1}=N_{2}=(G-1) / 4 \\
M_{2}=(G+3) / 4 \text { or } M_{1}=M_{2} \\
=N_{1}=(G-1) / 4, N_{2}=(G+3) / 4\end{array}$ & $\left(G^{4}+8 G^{3}+34 G^{2}+64 G+85\right) / 64$ \\
\hline$G=4 k+2$ & $\begin{array}{l}M_{1}=N_{1}=(G-2) / 4 \\
M_{2}=N_{2}=(G+2) / 4\end{array}$ & $\left(G^{4}+8 G^{3}+40 G^{2}+96 G+114\right) / 64$ \\
\hline$G=4 k+3$ & $\begin{array}{l}M_{1}=M_{2}=N_{2}=(G+1) / 4 \\
N_{2}=(G-3) / 4 \text { or } M_{1}=(G-3) / 4 \\
M_{2}=N_{1}=N_{2}=(G+1) / 4\end{array}$ & $\left(G^{4}+8 G^{3}+34 G^{2}+80 G+117\right) / 64$ \\
\hline
\end{tabular}
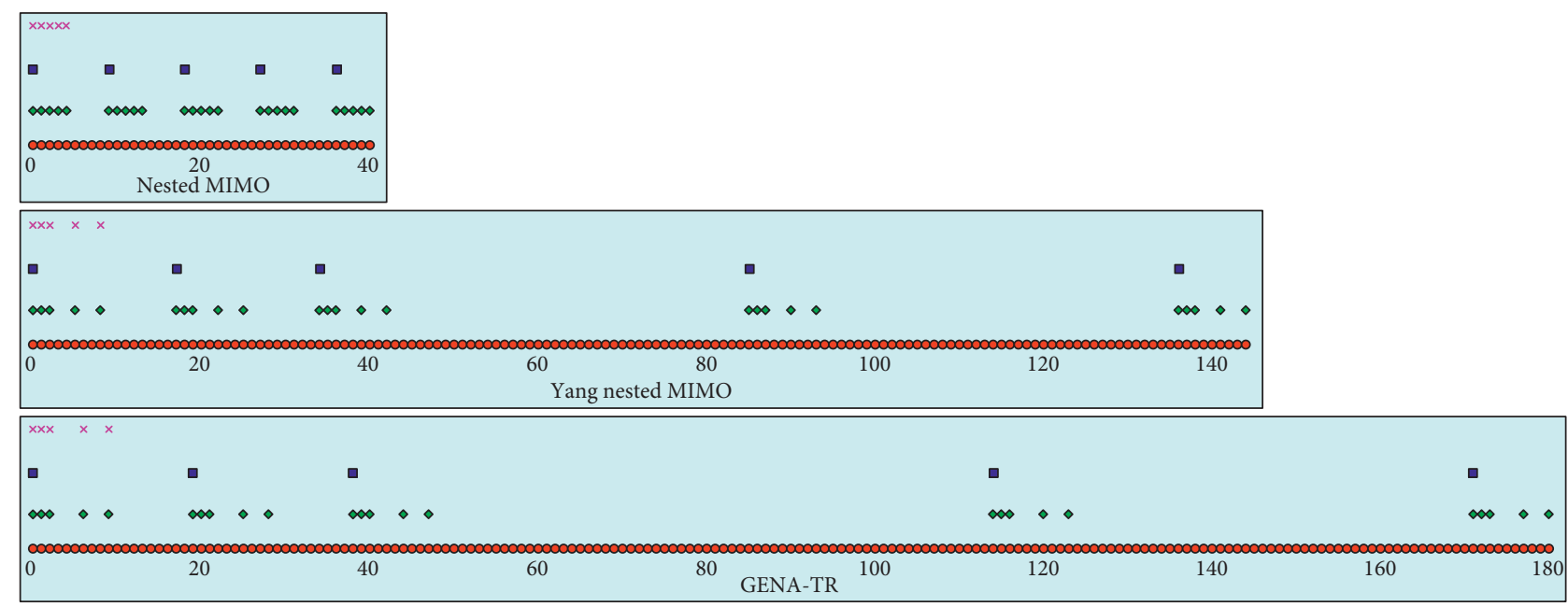

$\times$ Transmitter $\quad$ Sum coarray

- Receiver o Sum-difference coarray

Figure 3: An example of different MIMO radars with fiver transmitter and fiver receiver.

$$
\left\{\begin{array}{c}
2 M_{2}\left[2 N_{2}\left(N_{1}+1\right)+1\right]+\beta=0, \\
2\left(M_{1}+1\right)\left[2 N_{2}\left(N_{1}+1\right)+1\right]+\beta=0, \\
2 N_{2}\left[2 M_{2}\left(M_{1}+1\right)+1\right]+\beta=0, \\
2\left(N_{1}+1\right)\left[2 M_{2}\left(M_{1}+1\right)+1\right]+\beta=0, \\
M_{1}+M_{2}+N_{1}+N_{2-} G=0
\end{array}\right\} .
$$

By solving equation (22), the optimization results can be obtained, including the total number of physical sensors, the specific situation of the optimal array element configuration, and maximum DOF with the sum-difference coarray of generalized extended twolevel nested MIMO radar, as shown in Table 2.

To illustrate the distribution characteristics of the virtual array elements given by the proposition clearly, an example is shown in Figure 3, where $M_{1}=N_{1}=2, M_{2}=N_{2}=3$, $\alpha=2 M_{2}\left(M_{1}+1\right)+1=19$, and only the positive part is demonstrated due to the symmetry of SDCA. Moreover, nested MIMO [25] and Yang nested MIMO [27] are also given for comparison. It can be found that the consecutive lags of GENA-TR are $[0,180]$ in this example, which is higher than nested MIMO and Yang nested MIMO, and SDCA does not have holes.

Table 3 shows the consecutive lags and DOF of different MIMO geometries with a given total number of physical elements. It can be clearly seen that the GENA-TR not only retains the original advantages of the existing sparse array MIMO radar, whose sum-difference coarray is a hole-free ULA, but also significantly enhances DOF. Next, the redundant virtual array elements formed by sum-difference coarray can be averaged [30] and combined with the MUSIC algorithm based on Toeplitz matrix reconstruction for DOA estimation.

\section{Simulation Results}

In this section, several numerical simulations are presented to verify the rationality and superiority of the proposed nested MIMO radar (GENA-TR) and compare with other sparse array MIMO radars, including nested MIMO [25], NA-TR [26], Yang nested MIMO [27], Zheng nested MIMO [28], and ENA-TR. The total number of physical sensors is set as $G=10$. 
TABle 3: Consecutive lags and DOF of different MIMO radars.

\begin{tabular}{|c|c|c|c|c|c|c|}
\hline \multicolumn{2}{|c|}{ Nested MIMO } & \multicolumn{2}{|c|}{ Yang nested MIMO } & \multicolumn{2}{|c|}{ GENA-TR } & \multirow[b]{2}{*}{ DOF } \\
\hline Number of sensors & Consecutive lags & DOF & Consecutive lags & DOF & Consecutive lags & \\
\hline 10 & {$[-40,40]$} & 81 & {$[-144,144]$} & 289 & {$[-180,180]$} & 361 \\
\hline 13 & {$[-71,71]$} & 143 & {$[-356,356]$} & 713 & {$[-412,412]$} & 825 \\
\hline 17 & {$[-127,127]$} & 255 & {$[-955,955]$} & 1911 & {$[-1045,1045]$} & 2091 \\
\hline 22 & {$[-220,220]$} & 441 & {$[-2520,2520]$} & 5041 & {$[-2664,2664]$} & 5329 \\
\hline
\end{tabular}

5.1. DOF Comparison. In this numerical simulation, we compare the maximum DOF of GENA-TR with other nested MIMO geometries, where we change the total number of physical sensors from 10 to 20. It can be seen from Figure 4 that the maximum DOF of various nested MIMO radars increase with the number of physical array elements, and the proposed nested MIMO radar has the most obvious growth trend. In addition, it should be noted that when the number of physical elements is the same, the DOF of ENA-TR is only 4 higher than that of NA-TR, whereas GENA-TR can obtain a higher DOF than other MIMO radars.

5.2. Spatial Spectrum. In this numerical simulation, the Toeplitz matrix reconstruction based on the MUSIC algorithm is adopted to validate the spatial spectrum performance of GENA-TR and Yang nested MIMO radar [27], as shown in Figures 5 and 6. Suppose that there are $K=101$ far-field uncorrelated narrowband targets uniformly distributed from $-60^{\circ}$ to $60^{\circ}$ at an angular interval of $1.2^{\circ}$, where the signal-to-noise ratio (SNR) is equal to $10 \mathrm{~dB}$, the number of snapshots $L=1000$ and the search angel range is $\left[-90^{\circ}: 0.01^{\circ}: 90^{\circ}\right]$. It is obvious that GENA-TR can obtain better peaks and accurately estimate the DOA of all targets, whereas Yang nested MIMO radar has false peaks. Moreover, it is worth noting that the reason why NA-TR, ENA-TR, nested MIMO, and Zheng nested MIMO cannot estimate 101 targets is that MUSIC algorithm based on Toeplitz matrix reconstruction causes their DOF to be reduced by half, becoming $17,19,41$, and 81 , respectively.

5.3. Root Mean Square Error. In this numerical simulation, the root mean square error (RMSE) of DOA estimation for GENA-TR and other nested MIMO radars is compared via Monte Carlo experiments. It is assumed that there are $K=$ 13 far-field uncorrelated narrowband targets evenly distributed in $\left[-60^{\circ}: 10^{\circ}: 60^{\circ}\right]$. Figure 7 depicts the RMSE of different nested MIMO radars versus SNR, where the number of snapshots is $L=500$. Figure 8 shows the RMSE of different nested MIMO radars versus the number of snapshots, where SNR $=0 \mathrm{~dB}$. The RMSE of DOA estimation can be calculated as

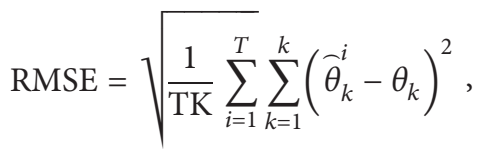

where $T=200$ represents the number of total ${ }_{i}$ Monte Carlo simulations, $\theta_{k}$ denotes the true DOA, and $\theta_{k}$ implies the estimated DOA of the $i$-th trials.

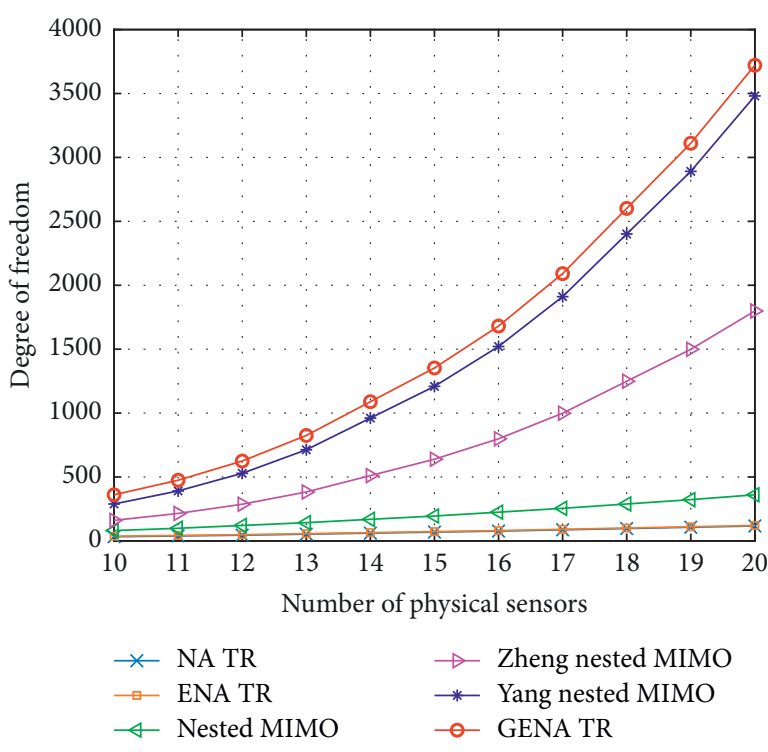

FIgURE 4: The maximum DOF of different nested MIMO versus the number of physical sensors.

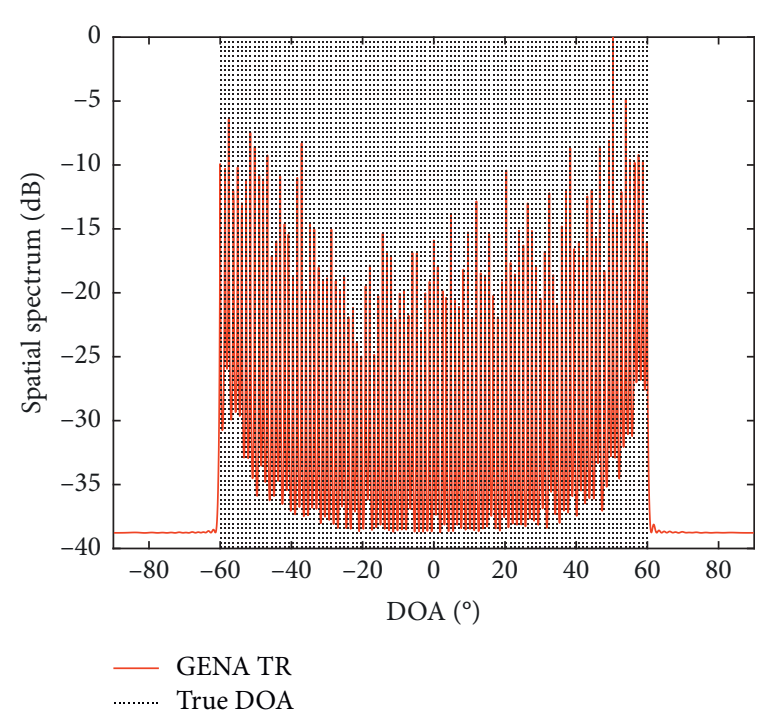

Figure 5: Spatial spectrum of GENA-TR.

It can be clearly seen from Figures 7 and 8 that as SNR and the number of snapshots increase, the DOA estimation accuracy of each MIMO radar has been improved, and simultaneously the DOA estimation performance of GENA- 


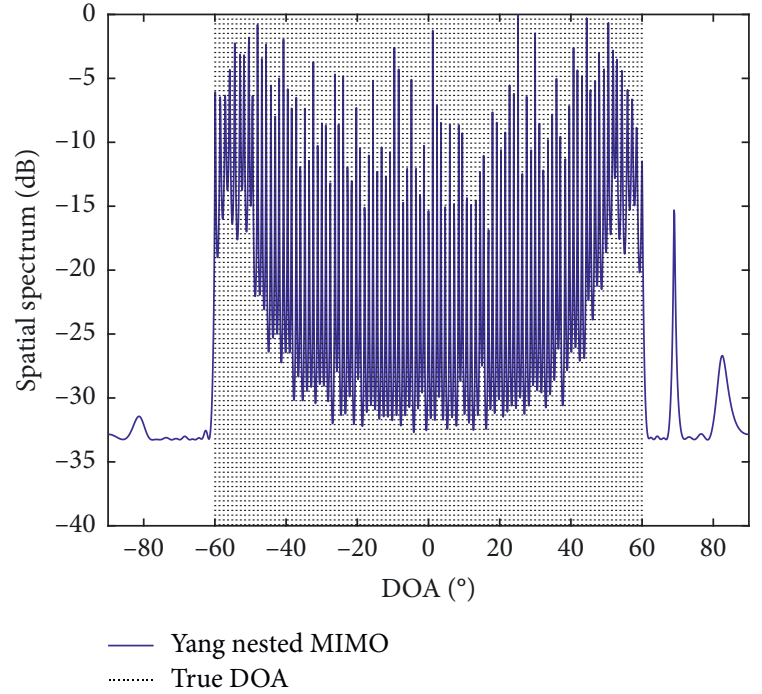

FIgURE 6: Spatial spectrum of Yang nested MIMO.

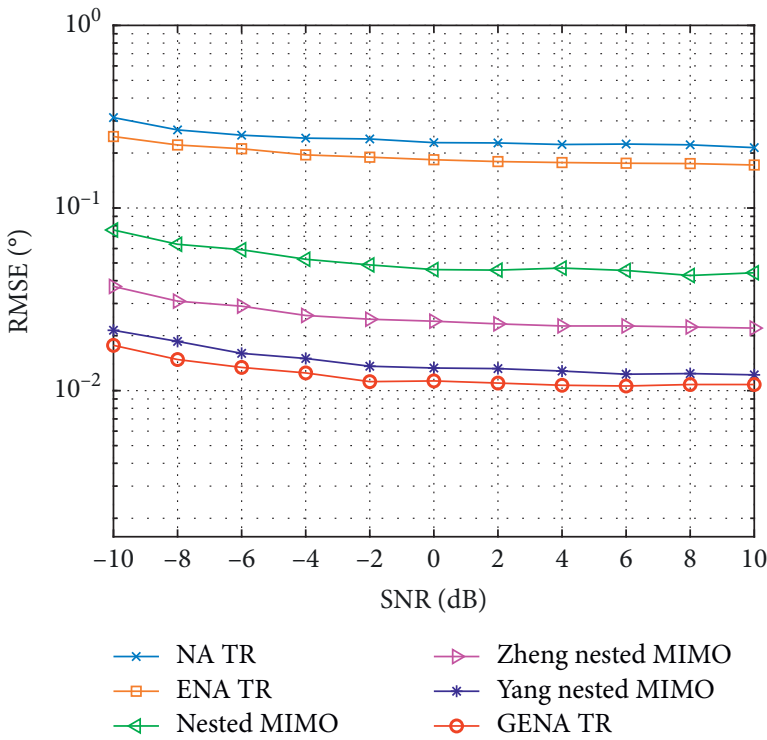

FIGURE 7: RMSE of different nested MIMO versus SNR.

TR is better than that of other MIMO radars. This is because the proposed GENA-TR can obtain a lager virtual element aperture when the number of physical sensors is the same.

5.4. Probability of Detection. In this numerical simulation, the probability of detection (PD) performance of DOA estimation with GENA-TR and other nested MIMO radars are compared via 200 Monte Carlo experiments. It is assumed that there are $K=13$ far-field uncorrelated narrowband targets evenly distributed in $\left[-60^{\circ}: 10^{\circ}: 60^{\circ}\right]$. Figure 9 shows the PD of different nested MIMO radars versus SNR, where the number of snapshots is $L=200$. Figure 10 shows the PD of different nested MIMO radars versus the number of snapshots, where $\mathrm{SNR}=-10 \mathrm{~dB}$. Here, $\mathrm{PD}$ is defined as the ratio of the number of numerical

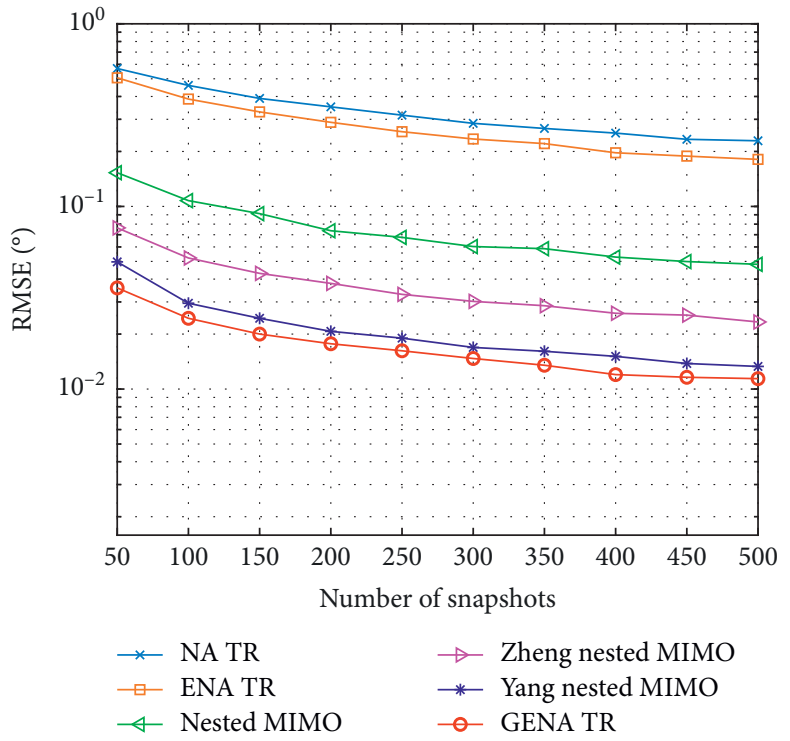

FIgURE 8: RMSE of different nested MIMO versus the number of snapshots.

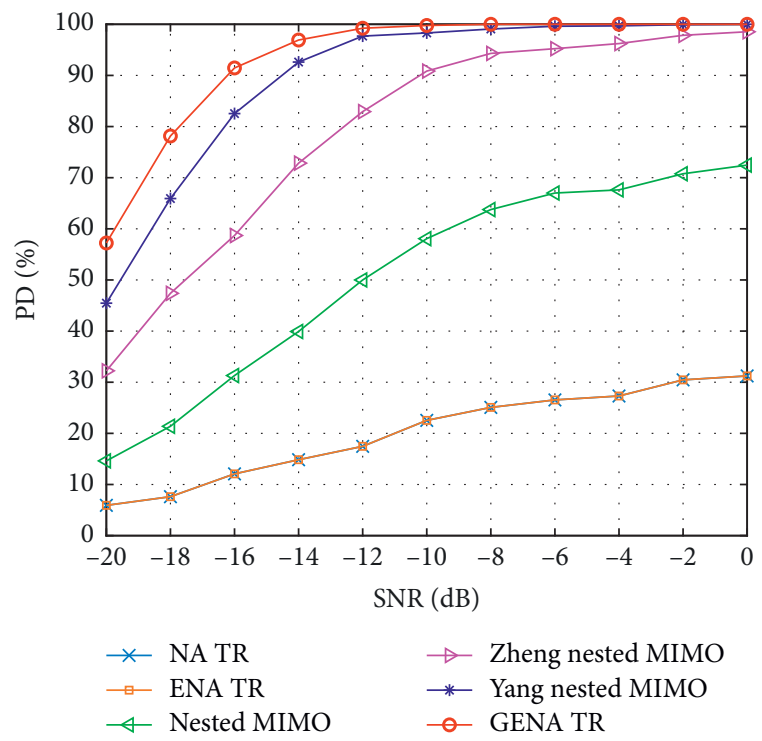

FIGURE 9: PD of different nested MIMO versus SNR.

simulations with the DOA estimation error within $\pm 0.01^{\circ}$ in the total experiments.

As shown in Figures 9 and 10, the PD of each nested MIMO radar has been improved as SNR and the number of snapshots increase. In addition, under the same SNR or the same number of snapshots, GENA-TR has a higher PD. Especially under the conditions of low SNR and low snapshots, the PD of the proposed nested MIMO radar is significantly better than that of other nested MIMO radars.

5.5. Resolution Performance. In this numerical simulation, the resolution performance of DOA estimation with GENATR and other nested MIMO radars is compared. Here, 


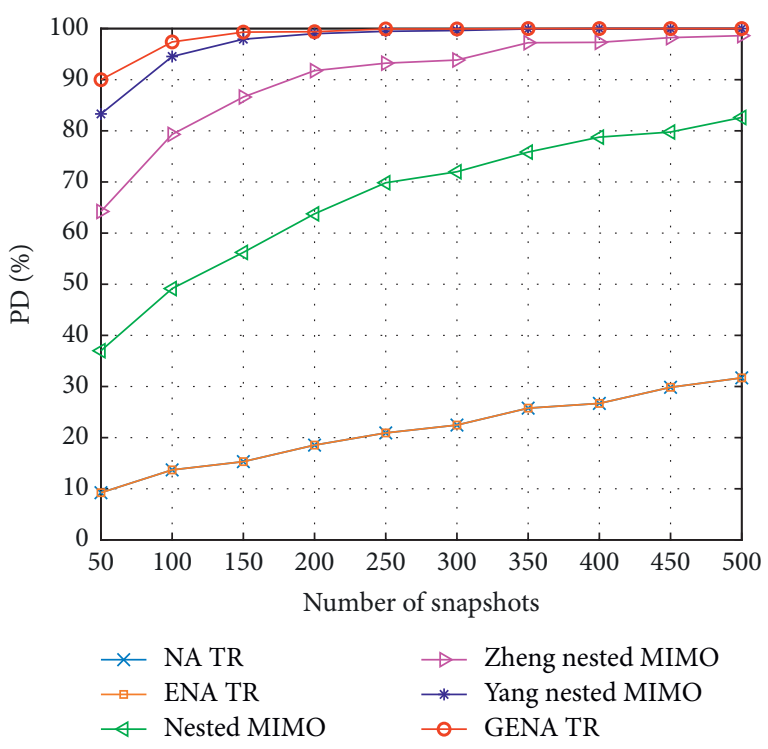

FIGURE 10: PD of different nested MIMO versus the number of snapshots.

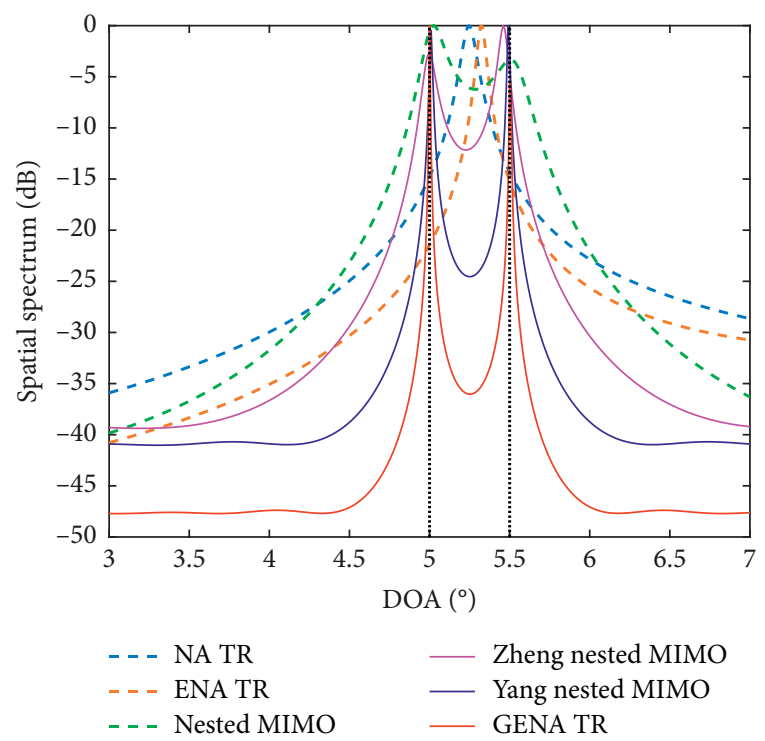

FIGURE 11: Spatial spectrum with two uncorrelated closely located targets.

resolution is defined as both the deviation between the true DOA and the estimated DOA of two closely targets which are less than one-half of the difference between the true angles of them [28]. Assume that there are $K=2$ far-field uncorrelated narrowband targets located at $\left[5^{\circ}, 5.5^{\circ}\right]$. Figure 11 demonstrates the spatial spectrum of the different nested MIMO radars, where $\mathrm{SNR}=-10 \mathrm{~dB}$ and the number of snapshots $L=200$.

It can be clearly seen from Figure 11 that NA-TR and ENA-TR cannot distinguish the above two targets, while other nested MIMO radars can distinguish the above two targets. Furthermore, GENA-TR has higher resolution and sharper peaks due to its higher degrees of freedom.

\section{Conclusions}

In this paper, a generalized extended two-level nested MIMO radar array configuration using extended two-level nested array is proposed, which can significantly improve the virtual array apertures and degrees of freedom, and the sum-difference coarray is a ULA without holes. In addition, the closed-form expressions of the maximum DOF are derived for a given number of physical sensors. At last, some numerical simulations are conducted to illustrate the advantages of the proposed GENA-TR in DOF, the estimation accuracy and the angle resolution.

\section{Data Availability}

The data supporting the conclusion of the article are shown in the research paper.

\section{Conflicts of Interest}

The authors declare that there are no conflicts of interest regarding the publication of this paper.

\section{Acknowledgments}

The authors would appreciate Chenghong Zhan and Zixin Zhang of Air and Missile Defense College, Air Force Engineering University, for their theoretical guidance and text correction. This work was supported by the Foundation of National Natural Science Foundation of China under Grant 61871395.

\section{References}

[1] E. Fishier, A. Haimovich, R. S. Blum et al., "MIMO radar: an idea whose time has come," in Proceedings of the IEEE Radar Conference, pp. 71-78, Philadelphia, PA, USA, April 2004.

[2] E. Jornson, L. Sanguinetti, H. Wymeersch et al., "Massive MIMO is a reality-what is next," Digital Signal Processing, vol. 94, pp. 3-20, 2019.

[3] P. Bezousek, P. Ezouske, and S. Karamazov, "MIMO radar signals with better correlation characteristics," Journal of Electrical Engineering, vol. 71, no. 3, pp. 210-216, 2020.

[4] T. Chen, J. Yang, and M. Guo, "A MIMO radar-based DOA estimation structure using compressive measurements," Sensors, vol. 19, no. 21, pp. 4706-4719, 2019.

[5] B. Liu, E. E. Kuruoglu, J. Zhang, F. Gini, T. Xue, and W. Lei, "Angle estimation in MIMO radar using a new sparse representation approach," International Journal of Electronics, vol. 106, no. 11, pp. 1694-1709, 2019.

[6] J. Du, M. Han, L. Jin, Y. Hua, and S. Li, "Target localization methods based on iterative super-resolution for bistatic MIMO radar," Electronics, vol. 9, no. 2, pp. 341-359, 2020.

[7] W. Zhang, W. Liu, J. Wang, and S. Wu, "Joint transmission and reception diversity smoothing for direction finding of coherent targets in MIMO radar," IEEE Journal of Selected Topics in Signal Processing, vol. 8, no. 1, pp. 115-124, 2014.

[8] E. Boudaher, F. Ahmad, M. G. Amin, and A. Hoorfar, "Mutual coupling effect and compensation in non-uniform arrays for direction-of-arrival estimation," Digital Signal Processing, vol. 61, pp. 3-14, 2017. 
[9] J. Konishi, H. Yamada, and Y. Yamaguchi, "Optimum element arrangements in MIMO radar using Khatri-Rao product virtual array processing," IEICE Communications Express, vol. 7, no. 11, pp. 407-414, 2018.

[10] A. Moffet, "Minimum-redundancy linear arrays," IEEE Transactions on Antennas and Propagation, vol. 16, no. 2, pp. $172-175,1968$.

[11] C. S. Ruf, "Numerical annealing of low-redundancy linear arrays," IEEE Transactions on Antennas and Propagation, vol. 41, no. 1, pp. 85-90, 1993.

[12] P. P. Vaidyanathan and P. Pal, "Sparse sensing with co-prime samplers and arrays," IEEE Transactions on Signal Processing, vol. 59, no. 2, pp. 573-586, 2011.

[13] P. Pal and P. P. Vaidyanathan, "Nested arrays: a novel approach to array processing with enhanced degrees of freedom," IEEE Transactions on Signal Processing, vol. 58, no. 8, pp. 4167-4181, 2010.

[14] M. Yang, L. Sun, X. Yuan, and B. Chen, "Improved nested array with hole-free DCA and more degrees of freedom," Electronics Letters, vol. 52, no. 25, pp. 2068-2070, 2016.

[15] Y. Iizuka and K. Ichige, "Extension of two-level nested array with larger aperture and more degrees of freedom," International Symposium on Antennas and Propagation, vol. 32, pp. 442-443, 2017.

[16] J. Liu, Y. Zhang, Y. Lu, S. Ren, and S. Cao, "Augmented nested arrays with enhanced DOF and reduced mutual coupling," IEEE Transactions on Signal Processing, vol. 65, no. 21, pp. 5549-5563, 2017.

[17] H. Huang, B. Liao, X. Wang, X. Guo, and J. Huang, "A new nested array configuration with increased degrees of freedom," IEEE Access, vol. 6, pp. 1490-1497, 2018.

[18] Y. Huang, G. Liao, J. Li, J. Li, and H. Wang, "Sum and difference coarray based MIMO radar array optimization with its application for DOA estimation," Multidimensional Systems and Signal Processing, vol. 28, no. 4, pp. 1183-1202, 2017.

[19] C. Chen and P. P. Vaidyanathan, "Minimum redundancy MIMO radars," Proceedings of the IEEE International Symposium on Circuits Systems (ISCAS), vol. 34, pp. 45-48, 2008.

[20] J. Li, D. Jiang, and X. Zhang, "DOA estimation based on combined unitary ESPRIT for coprime MIMO radar," IEEE Communications Letters, vol. 21, no. 1, pp. 96-99, 2017.

[21] E. Boudaher, F. Ahmad, and M. G. Amin, "Sparsity-based direction finding of coherent and uncorrelated targets using active nonuniform arrays," IEEE Signal Processing Letters, vol. 22, no. 10, pp. 1628-1632, 2015.

[22] J. Shi, G. Hu, X. F. Sun, W. Zheng, and Y. Xiao, "Generalized Co-prime MIMO radar for DOA estimation with enhanced degrees of freedom," IEEE Sensors Journal, vol. 18, no. 3, pp. 1203-1212, 2018.

[23] R. Zhang, "Multiple emitter location and signal parameter estimation," IEEE Transactions on Antennas and Propagation, vol. 34, no. 3, pp. 276-280, 1986.

[24] R. Roy and T. Kailath, "ESPRIT-estimation of signal parameters via rotational invariance techniques," IEEE Transactions on Acoustics, Speech, and Signal Processing, vol. 37, no. 7, pp. 984-995, 1989.

[25] S. Qin, Y. D. Zhang, and M. G. Amin, "DOA estimation of mixed coherent and uncorrelated signals exploiting a nested MIMO radar," Proceedings of the of IEEE Benjamin Franklin Symposium on Microwave and Antenna Sub-systems for Radar, vol. 81, 2016.

[26] G. Zheng and J. Tang, "DOD and DOA estimation in bistatic MIMO radar for nested and coprime array with closed-form
DOF," International Journal of Electronics, vol. 104, no. 5, pp. 885-897, 2016.

[27] M. Yang, L. Sun, X. Yuan, and B. Chen, "A new nested MIMO array with increased degrees of freedom and hole-free difference coarray," IEEE Signal Processing Letters, vol. 25, no. 1, pp. 40-44, 2018.

[28] W. Zheng, X. Zhang, and J. Shi, "Sparse extension array geometry for DOA estimation with nested MIMO radar," IEEE Access, vol. 5, pp. 9580-9586, 2017.

[29] W. He, X. Yang, and Y. D. Wang, "A high-resolution and lowcomplexity DOA estimation method with unfolded coprime linear arrays," Sensors, vol. 20, no. 1, pp. 218, 2020.

[30] C. H. Zhan, G. P. Hu, Z. X. Zhang et al., "Increasing utilization of redundant virtual array for DOA estimation based on coprime array," Mathematical Problems in Engineering, vol. 12, 2020 